\title{
Interference in Exclusive Vector Meson Production in Heavy Ion Collisions
}

\author{
Spencer R. Klein and Joakim Nystrand \\ Lawrence Berkeley National Laboratory, Berkeley, CA 94720
}

\begin{abstract}
Photons emitted from the electromagnetic fields of relativistic heavy ions can fluctuate into quark anti-quark pairs and scatter from a target nucleus, emerging as vector mesons. These coherent interactions are identifiable by final states consisting of the two nuclei and a vector meson with a small transverse momentum. The emitters and targets can switch roles, and the two possibilities are indistinguishable, so interference may occur. Vector mesons are negative parity so the amplitudes have opposite signs. When the meson transverse wavelength is larger than the impact parameter, the interference is large and destructive.

The short-lived vector mesons decay before amplitudes from the two sources can overlap, and so cannot interfere directly. However, the decay products are emitted in an entangled state, and the interference depends on observing the complete final state. The non-local wave function is an example of the Einstein-Podolsky-Rosen paradox.
\end{abstract}


In relativistic heavy ion collisions, vector mesons are copiously produced via photonPomeron fusion [1]. The photon and Pomeron both couple coherently to their emitting nuclei, giving these reactions a distinctive signature, with the final state consisting of the two nuclei, a vector meson with low perpendicular momentum $\left(p_{\perp}\right)$ and nothing else. The latter requirement restricts these interactions to peripheral collisions, usually with impact parameter $b>2 R_{A}, R_{A}$ being the nuclear radius. At heavy ion colliders, vector mesons with masses up to about $2 \gamma \hbar c / R_{A}$, where $\gamma$ is the Lorentz boost of each beam are produced.

The electromagnetic field has a long range, while the nuclear (Pomeron) field has a short range compared to the size of the nucleus. So, vector meson production occurs in the region occupied by the Pomeron emitting ('target') nucleus. Since the production is coherent over the entire target nucleus, it is a fairly good approximation to treat the meson production regions as two point sources, one at the center of each nucleus. The situation is similar to that in a two-source interferometer, albeit with unstable particles. A parity inversion switches the emitter and target. Because of the vector meson negative parity, the two emission amplitudes have opposite sign. For perpendicular momentum $p_{\perp} \ll 1 / b, b$ being the impact parameter, the interference is destructive. We calculate here the magnitude of the interference and discuss the implications of the short vector meson lifetime, which causes the mesons to decay before wave functions from the two sources can overlap.

The cross sections for meson production can be calculated by convoluting the WeizsäckerWilliams virtual photon spectrum with the photonuclear interaction cross section. The photonuclear cross section is determined from data on $\gamma p$ interactions [2], using a Glauber formalism to find the cross section for $\gamma A \rightarrow V A$, where $A$ is the target nucleus, and $\mathrm{V}$ a vector meson. In this approach, the photons are considered to fluctuate to virtual vector mesons, which then elastically scatter from the target nucleus, emerging as real mesons.

Earlier calculations of production cross sections summed the cross sections from the two nuclei and found very large cross sections [1]. Exclusive $\rho^{0}$ production was about $10 \%$ of the hadronic cross section for gold on gold collisions at a per nucleon center of mass energy $\sqrt{S_{N N}}=200 \mathrm{GeV}$ as will be studied at the Relativistic Heavy Ion Collider (RHIC), rising 
to $30 \%$ for the $\sqrt{S_{N N}}=5.5 \mathrm{TeV}$ lead on lead collisions at the Large Hadron Collider (LHC). The $\omega$ and $\phi$ production are about an order of magnitude smaller, with $J / \psi$ cross sections of $0.3 \mathrm{mb}$ (gold at RHIC) up to $32 \mathrm{mb}$ (Pb at LHC). These cross sections correspond to production of hundreds of $\rho^{0}$ per second at RHIC, and hundreds of thousand of $\rho^{0}$ per second with calcium beams at the LHC. The $J / \psi$ rates range from $0.06 \mathrm{~Hz}$ with gold at RHIC up to $780 \mathrm{~Hz}$ with calcium at LHC. These rates are comparable to those found at current and future meson factories.

Because of the small $b$, for low $p_{\perp}$ final states it is impossible to tell which nucleus emitted the photon, and which elastically scattered the meson. So, the amplitudes are combined. Because vector mesons are negative parity, the two amplitudes subtract, rather than add, leading to destructive interference. In different terms, for $p_{\perp} b<1$, a system of two identical nuclei has zero dipole moment, so radiation of vector particles is impossible. This situation is analogous to bremsstrahlung by identical particles [3], where there can be almost complete destructive interference. However, in contrast to bremsstrahlung photons, vector mesons are short lived, and they don't live long enough to travel the distance $b$. So, the vector mesons generally decay before their wave functions can overlap. However, their decay products can interfere.

The amplitude for vector meson production is $A\left(y, p_{\perp}, b\right) e^{i \phi(y)}$, with $A\left(y, p_{\perp}, b\right)$ the magnitude and $\phi(y)$ the phase at rapidity $y$. We assume that $A\left(y, p_{\perp}, b\right) e^{i \phi(y)}$ is symmetric to rotations around the beam direction. The photon energy and perpendicular momentum are $k$ and $k_{\perp}$, and the final meson momentum $p$. All values are in the center of mass frame, which corresponds to the laboratory frame for a heavy ion collider. To eliminate the directional ambiguity, we adopt the convention that, for $y>0$, the photon energy is higher than the Pomeron energy. Then, $A(y>0)<A(y<0)$ and $y=\ln \left(2 k / M_{V}\right)$. These amplitudes are determined from the cross sections calculated in Ref. [1], using $A\left(y, p_{\perp}, b\right)=\sqrt{\sigma_{A+A \rightarrow A+A+V}\left(y, p_{\perp}, b\right)}$, for a single production direction.

The final state perpendicular momentum is the sum of the photon and Pomeron per- 
pendicular momentum; the spectrum is a convolution of the two sources. The photon perpendicular momentum can be found with the equivalent photon approximation [4]:

$$
\frac{d^{3} N_{\gamma}\left(k, k_{\perp}\right)}{d^{2} k_{\perp} d k}=\frac{\alpha^{2} Z^{2} F^{2}\left(k_{\perp}^{2}+k^{2} / \gamma^{2}\right) k_{\perp}^{2}}{\pi^{2}\left(k_{\perp}^{2}+k^{2} / \gamma^{2}\right)^{2}} .
$$

The nuclear form factor,

$$
F(q)=\frac{4 \pi \rho_{0}}{A q^{3}}\left[\sin \left(q R_{A}\right)-q R_{A} \cos \left(q R_{A}\right)\right]\left[\frac{1}{1+a^{2} q^{2}}\right]
$$

the convolution of a hard sphere and a Yukawa potential with range $a=0.7 \mathrm{fm}$, is an excellent fit to a Woods-Saxon density distribution [1]. Here, $\rho_{0}$ is the nuclear density and $A$ the atomic number. For a given $k, k_{\perp}$ is independent of $b$, with $d^{3} N_{\gamma}\left(k, k_{\perp}\right) / d^{2} k_{\perp} d k$ rising from 0 at $k_{\perp}=0$ to a maximum at $k_{\perp}=k / \gamma$ and then dropping as $k_{\perp}$ rises further.

Since the Pomeron range is short, its perpendicular momentum spectrum is determined by Eq. (2). Figure 1 shows the photon and Pomeron perpendicular momentum spectra, along with their convolution, for $y=0$, and $y=-2$. Diffractive fringes are visible in the Pomeron spectrum; since the photon contribution to $p_{\perp}$ is usually small, these fringes also appear in the final $p_{\perp}$ spectrum.

The production phase depends on the process. Since the soft Pomeron represents the absorptive part of the cross section, the amplitude for photon-Pomeron fusion should be largely imaginary, with a small real part because the cross section rises slowly with photon energy [5]. Parameterizing $\sigma(\gamma N \rightarrow V N) \sim W^{\epsilon}$, where $W$ is the $\gamma$-nucleon center of mass energy, $\phi(y)=\tan ^{-1}(\pi \epsilon / 4)$, independent of $y[6]$. For the light mesons, $\epsilon=0.22$ so $\phi \sim 10^{0}$. For the $J / \psi, \sigma(\gamma N \rightarrow V N) \sim W^{0.8}$, inconsistent with the soft Pomeron; the steep rise may be due to threshold behavior or signal the breakdown of the soft Pomeron model [7]; in either case, the $J / \psi$ phase angle must be treated with caution.

For the $\rho^{0}$ and $\omega$, photon-meson (primarily the $f_{0}$ [0]) fusion also contributes to the cross section, which may be parameterized as $X W^{\epsilon}+Y W^{-\eta}$, where $\eta>1$, so the photon-meson contribution decreases as $W$ rises. Besides increasing the cross section, this causes $\phi(y)$ to vary with $y$. Because the phase has only been measured as an average over photon energy 
[8], we will treat $\phi(y)$ as a constant. Of course, at $y=0$, the energies are equal; as $|y|$ rises, the phase difference could become significant.

For two nuclei at points $\overrightarrow{x_{1}}$ and $\overrightarrow{x_{2}}$, the amplitude $A_{0}$ for observing a vector meson at a distant point $x_{0}$ is found by approximating the vector mesons by plane waves. The meson momentum $\vec{p}$ is determined by $p_{\perp}$ and $k$ via $p_{\|}=k-m_{V}^{2} / 4 k$.

$$
A_{0}\left(x_{0}, \vec{p}, b\right)=A\left(p_{\perp}, y, b\right) e^{i\left[\phi(y)+\overrightarrow{p^{\prime}} \cdot\left(\overrightarrow{x_{1}}-\overrightarrow{x_{0}}\right)\right]}-A\left(p_{\perp},-y, b\right) e^{i\left[\phi(-y)+\overrightarrow{p^{\prime}} \cdot\left(\overrightarrow{x_{2}}-\overrightarrow{x_{0}}\right)\right]}
$$

with the negative sign because of the negative parity. Because of their short lifetimes, the vector mesons will decay before reaching $x_{0}$. In fact, they will generally decay before their wave functions can overlap. However, their decay products can interfere; with $\vec{p}$ now being the sum of the product momenta. The resulting virtual interference pattern depends only on $\vec{p} \cdot \vec{b}$. The decay is incorporated by multiplying $A\left(p_{\perp}, y, b\right)$ by the decay amplitude, including the branching ratios, lifetime and angular dependence. Since the angular distributions are the same for the two production directions, the decay amplitudes are the same for both directions, and does not affect the observed cross sections.

Since the decay products fly off in different directions at relativistic velocities, the production amplitudes from the two sources can only interfere if the final state wave function is nonlocal [9]. For example, in $J / \psi \rightarrow e^{+} e^{-}$, the electron and positron are nearly back-toback, and the $e^{+}$(or $e^{-}$) amplitudes from the two sources cannot overlap each other until they are a good distance from their parent $J / \psi$. By the time the $e^{+}$(and $e^{-}$) from the two sources overlap, the $e^{+}$and $e^{-}$are well separated, and any interference involving both the $e^{+}$and $e^{-}$requires a non-local wave function.

The final state wave function is entangled, with the form $\exp \left(i k_{1} \cdot x_{1}\right) \exp \left(i k_{2} \cdot x_{1}\right)-\exp \left(i k_{2}\right.$. $\left.x_{2}\right) \exp \left(i k_{2} \cdot x_{2}\right)$ where $k_{1}$ and $k_{2}$ are the individual decay product momenta (for a 2 body decay), and cannot be factored into individual particle wave functions. This interference is visible by observing the complete final state, but not by examining the individual decay products. This non-locality is an example of the Einstein-Podolsky-Rosen paradox [10]. However, the correlation depends on $p_{\perp}$, which is a continuous variable, rather than a 
discrete variable like spin, as is commonly considered.

The individual decay products have much higher momenta than their parent meson, and in principle could be used to reconstruct the meson decay point, and hence determine which nucleus emitted the meson. However, the position measurement would obscure their momentum enough to eliminate the interference pattern, as occurs with a two-slit interferometer [11].

In the center of mass frame, production is nearly simultaneous (within $\Delta t=b / c \gamma$ ), so time drops out. Since $A$ is defined to be real,

$\sigma\left(p_{\perp}, y, b\right)=A^{2}\left(p_{\perp}, y, b\right)+A^{2}\left(p_{\perp},-y, b\right)-2 A\left(p_{\perp}, y, b\right) A\left(p_{\perp},-y, b\right) \cos [\phi(y)-\phi(-y)+\vec{p} \cdot \vec{b}]$

At midrapidity, $y=0$, the two source contributions are equal and the observed cross section is

$$
\sigma\left(p_{\perp}, y=0, b\right)=2 A^{2}\left(p_{\perp}, y=0, b\right)(1-\cos [\vec{p} \cdot \vec{b}])
$$

For a given $b, \sigma$ oscillates with period $\Delta p_{\perp}=\hbar / b$. When $\vec{p} \cdot \vec{b} \ll 1$, the interference is destructive, and there is little emission.

Since $b$ is in principle measurable by examining the outgoing ions, it is an observable, and we integrate the cross section (not amplitude) over all possible $b$ to get the total production. Figure 2 shows $d^{2} N / d p_{\perp}^{2}$ at $y=0$ for gold and silicon beams at RHIC and calcium at LHC, with and without interference.

In theory, one could choose to measure the perpendicular momentum transfer from the two nuclei instead of $b$. Then, for roughly $p_{\perp}>10 \mathrm{MeV} / \mathrm{c}$, the larger momentum transfer will usually come from the target. This determination will break the symmetry, reducing the magnitude of the interference. Of course, near $p_{\perp}=0$, the photon and Pomeron perpendicular momenta have very similar magnitudes, so the interference will remain. Because the initial nuclear momenta are not well known, this possibility seems at best theoretical.

With gold beams at RHIC, vector meson production occurs with median impact parameters for $\rho^{0}, \omega, \phi$ and $J / \psi$ production of $46 \mathrm{fm}, 46 \mathrm{fm}, 38 \mathrm{fm}$ and $23 \mathrm{fm}$ respectively, 
corresponding to $p_{\perp}<4 \mathrm{MeV} / \mathrm{c}$ for the light mesons and $p_{\perp}<10 \mathrm{MeV} / \mathrm{c}$ for the $J / \psi$. Below these $p_{\perp}$ values, interference is large. With lighter nuclei, the average impact parameters are slightly smaller, leading to higher momentum cutoffs. At the LHC, impact parameters are much larger, 170-290 fm for light vector mesons and 44-68 fm for the $J / \psi$, and the reduction is large only for quite small values of $p_{\perp}$.

Despite the dramatically different $p_{\perp}$ spectrum, the the overall rate at $y=0$ is unaffected by the interference. This is because $\left\langle p_{\perp}\right\rangle>\langle\hbar / b\rangle$, so the $\cos [\vec{p} \cdot \vec{b}]$ oscillations average out, leaving the rate almost unaffected. Since the $p_{\perp}$ spectrum is almost independent of $y$, this remains true at other rapidities, so the total cross section is also unchanged.

Figure 3 shows $d^{2} N / d p_{\perp}^{2}$ at $y=1$ for the same three cases. The magnitude of the interference is reduced because of the different amplitudes for the two directions. Besides the overall amplitudes, the spectrum is slightly different, because the photons have different energies, and so the $p_{\perp}$ spectra are slightly different.

This interference can be studied with the decays $\rho^{0} \rightarrow \pi^{+} \pi^{-}, \omega \rightarrow \pi^{+} \pi^{-}, \phi \rightarrow K^{+} K^{-}$ and $J / \psi \rightarrow e^{+} e^{-}$which are readily reconstructible. Because of the high rates, the backgrounds to these processes should be small; simulations indicate that this is indeed the case, at least for a large acceptance detector [12].

There are a few details that may slightly reduce the interference. Because the photon emission is electromagnetic, it is possible for the emitting nucleus to be excited into a giant dipole resonance (GDR); since the Pomeron is uncharged, excitation is unlikely for the scattering nucleus. However, even for the photon emitting nucleus, the excitation probability is relatively low. Excitation is much more likely if the nuclei exchange an additional photon between themselves, with equal excitation probabilities for both nuclei, and not affecting the interference [13]. Radiative corrections and other higher order processes could also reduce the interference. These factors should be small because the nuclear trajectories are barely affected by the interaction. Likewise, backgrounds from non-resonant (but coherent) photonuclear and two-photon processes should be small.

However, these backgrounds may introduce additional interference terms which may 
complicate the picture. For example, the process $\gamma \gamma \rightarrow e^{+} e^{-}$can interfere with $e^{+} e^{-}$from $J / \psi$ decays; the rate for the $\gamma \gamma \rightarrow e^{+} e^{-}$to mimic $J / \psi$ decays is small, but perhaps visible. In conclusion, interference between emission between two colliding nuclei dramatically changes the perpendicular momentum spectrum of exclusively produced vector mesons, suppressing production of vector mesons at low $p_{\perp}$. Because the vector mesons decay before their wave functions can overlap, the interference involves all of the decay products, which never overlap with each other. So, the decay products have an entangled, non-local wave function.

We would like to acknowledge useful conversations with Stan Brodsky and Jørgen Randrup. This work was supported by the US DOE, under contract DE-AC-03-76SF00098. 


\section{REFERENCES}

[1] S. Klein and J. Nystrand, Phys. Rev. C60, 014903 (1999).

[2] These calculations are based on fits in J. A. Crittenden, Exclusive Production of Neutral Vector Mesons at the Electron-Proton Collider HERA (Springer-Verlag, Berlin, 1997). Similar, but slightly newer results appear in J. Breitweg et al., Z. Phys. C75, 215 (1997) and S. Aid et al., Nucl. Phys. B472, 3 (1996).

[3] R. C. Stabler, Nature 206, 922 (1965).

[4] M. Vidovic, M. Greiner, C. Best and G. Soff, Phys. Rev. C47, 2308 (1993); G. Baur and L. G. Ferreira Filho, Phys. Lett. B254, 30 (1991).

[5] D. Leith in Electromagnetic Interactions of Hadrons, ed. A. Donnachie and G. Shaw, Plenum Press, 1978, and references therein.

[6] J. R. Forshaw and D. A. Ross, Quantum Chromodynamics and the Pomeron, Cambridge, 1997.

[7] N. Cartiglia, hep-ph/9703245, Mar., 1997.

[8] T. H. Bauer et al., Rev. Mod. Phys. 50, 261 (1978).

[9] M. A. Horne, A. Shimony and A. Zeilinger, Phys. Rev. Lett. 62, 2209 (1989).

[10] A. Einstein, B. Podolsky and N. Rosen, Phys. Rev. 47, 777 (1935).

[11] This situation is analogous to the two-slit interferometer described by T. Sudbery, in Quantum Concepts in Space and Time, ed. R. Penrose and C. J. Isham, (Oxford, 1986).

[12] J. Nystrand and S. Klein, nucl-ex/9811007, in Proc. Workshop on Photon Interactions and the Photon Structure, Lund, Sweden, Sept., 1998; S. Klein and J. Nystrand, STAR Note 347, June, 1998. Available on the web at http://www.star.bnl.gov/star/starlib/doc/www/sno/ice/sn0347.htm. 
[13] G. Baur, K. Hencken and D. Trautman, J. Phys. G 24, 1657 (1998). 


\section{FIGURES}

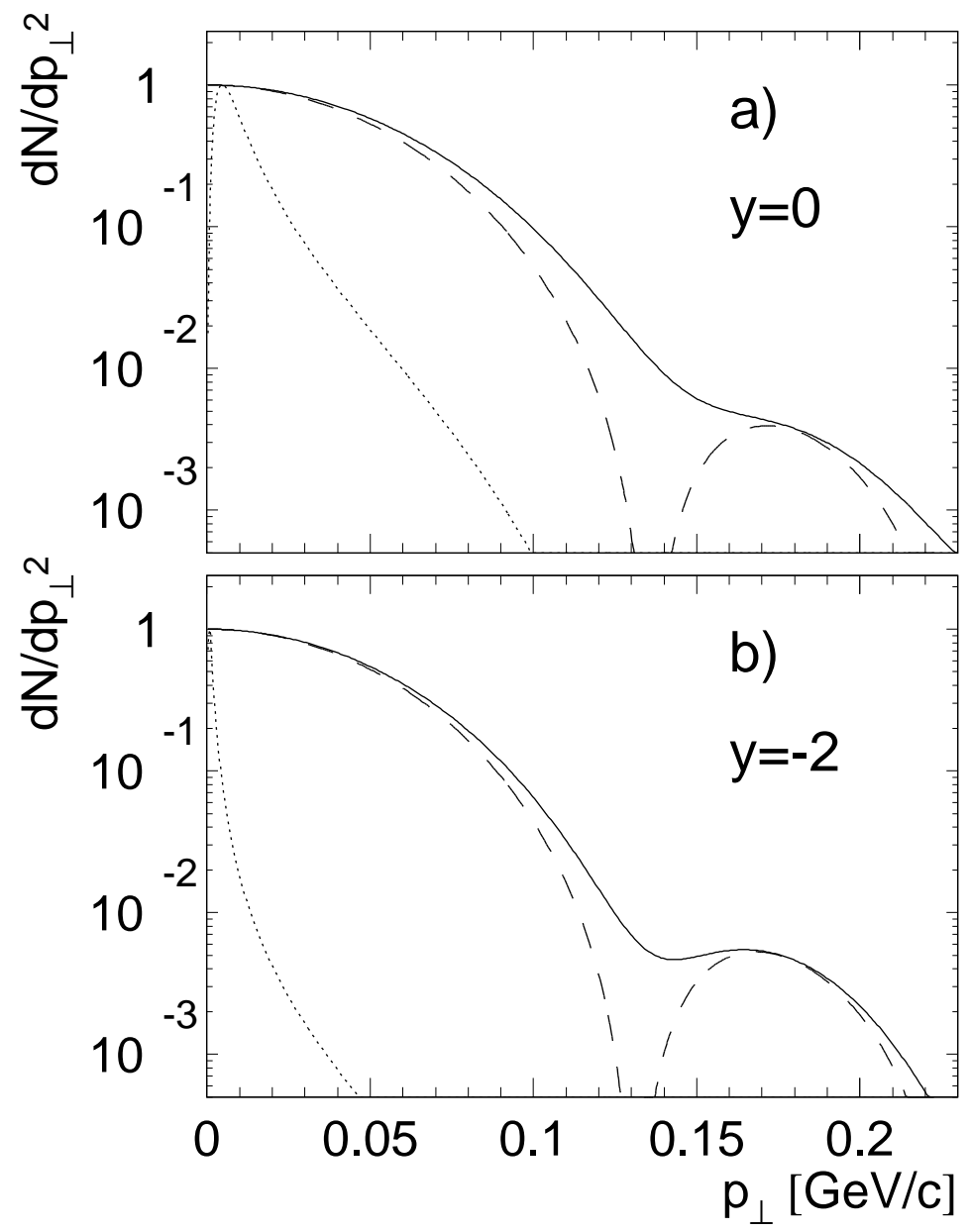

FIG. 1. Perpendicular momentum spectra for photons (dotted curves), Pomerons (dashed curves) and the final state vector mesons (solid curves) at (a) $y=0$, and (b) $y=-2$ (corresponding to $k=69 \mathrm{MeV}$ in the lab frame) for $\phi$ production in gold collisions at RHIC. The curves are each normalized to a maximum $d N / d p_{\perp}^{2}=1$. Clear diffraction minima appear in the Pomeron spectra. Since $k_{\perp}$ is small compared to $p_{\perp}$, the minima remain visible in the $\phi p_{\perp}$ spectrum. 


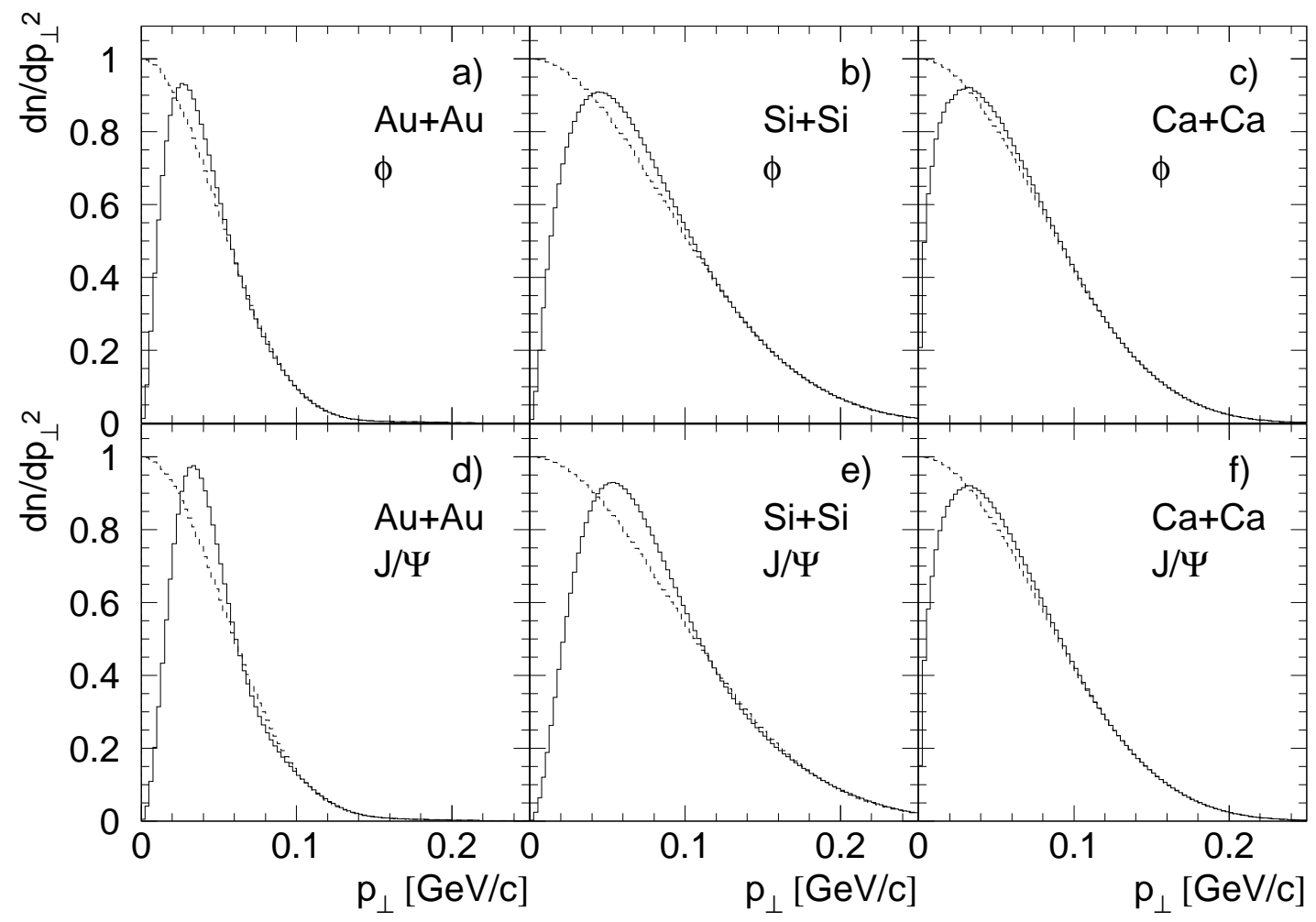

FIG. 2. Expected $p_{\perp}$ spectra for reconstructed $\phi$ and $J / \psi$ mesons at $\mathrm{y}=0$ with (a,d) gold beams at RHIC, (b,e) silicon beams at RHIC and (c,f) calcium beams at LHC. The solid histograms include interference, while the dotted ones do not. Because of the smaller impact parameters production is peaked at higher $p_{\perp}$ in (b,e) than in (a,d). Because of the smaller impact parameters in (b,e), the interference dip extends to higher $p_{\perp}$ than in $(\mathrm{a}, \mathrm{d})$. In $(\mathrm{c}, \mathrm{f})$, the energies are higher, leading to higher impact parameters, pushing the interference dip to lower $p_{\perp}$. The figure is normalized so that, without interference $d n / d p_{\perp}^{2}=1$ at $p_{\perp}=0$; the rates are given in Ref. [1]. 


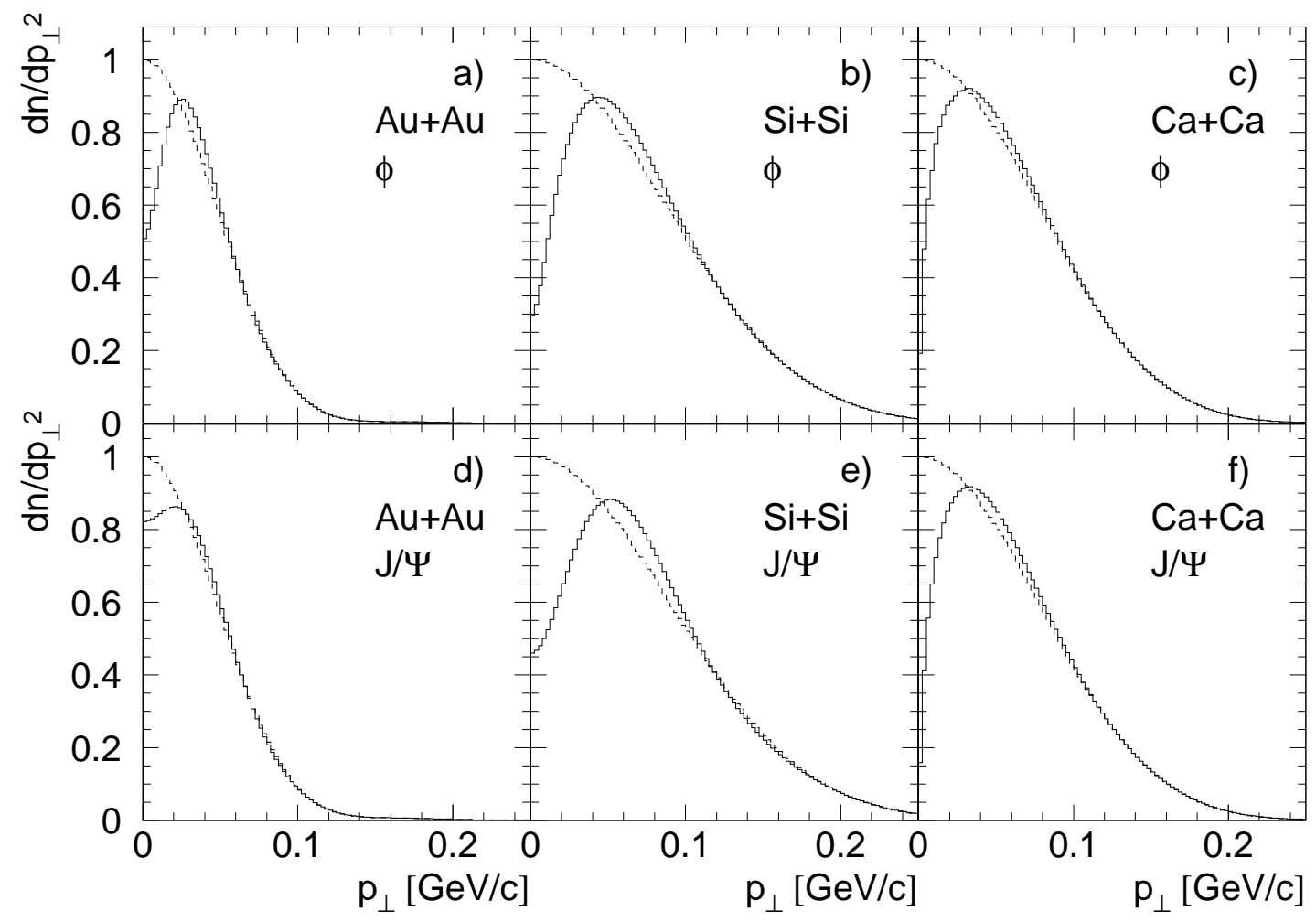

FIG. 3. Expected $p_{\perp}$ spectra for reconstructed $\phi$ and $J / \psi$ mesons at $\mathrm{y}=1$ with (a,d) gold beams at RHIC, (b,e) silicon beams at RHIC and (c,f) calcium beams at LHC. The solid histograms include interference, while the dotted ones do not. Because of the difference in amplitudes, $d N / d p_{\perp}^{2} \neq 0$ at $p_{\perp}=0$. The size of the dip at $p_{\perp}=0$ depends on the ratio of amplitudes at $y=1$ and $y=-1$; it is larger for lighter nuclei and higher collision energies. This figure is normalized so that, without interference $d n / d p_{\perp}^{2}=1$ at $p_{\perp}=0$; the rates are given in Ref. [1]. 